\title{
A 96 CHANNEL RECEIVER FOR THE ILCTA LLRF SYSTEM AT FERMILAB*
}

\author{
Uros Mavric ${ }^{\#}$, Brian Chase, Julien Branlard, Ed Cullerton, Dan Klepec \\ Fermi National Accelerator Laboratory, Batavia, IL, USA.
}

\begin{abstract}
The present configuration of an ILC main LINAC RF station has 26 nine cell cavities driven from one klystron. With the addition of waveguide power coupler monitors, 96 RF signals will be down-converted and processed. A down-converter chassis is being developed that contains 12 eight channel analog modules and a single upconverter module. This chassis will first be deployed for testing a cryomodule composed of eight cavities located at New Muon Laboratory (NML) - Fermilab. Critical parts of the design for LLRF applications are identified and a detailed description of the circuit with various characteristic measurements is presented. The board is composed of an input band-pass filter centered at $1.3 \mathrm{GHz}$, followed by a mixer, which down-converts the cavity probe signal to a proposed $13 \mathrm{MHz}$ intermediate frequency. Cables with 8 channels per connector and good isolation between channels are being used to interconnect each down-converter module with a digital board. As mixers, amplifiers and power splitters are the most sensitive parts for noise, nonlinearities and crosstalk issues, special attention is given to these parts in the design of the LO port multiplication and distribution.
\end{abstract}

\section{INTRODUCTION}

Identifying the main error contributors in the LLRF electronics is important for error minimization. The final performance of the receiver is defined by many phenomena, which makes the error identification problem more complex. Usually a combination of computer simulations and prototype on-the-bench testing usually helps to understand the major pitfalls. A Matlab based model has been developed to study the impact of the receiver parameters on the control of multiple cavities.

\section{Functional Block Diagram of the Receiver}

The 96 channels receiver will be organized in 8 channels per board, which requires 12 boards. The size of the board and consequently the number of channels per board was chosen to facilitate the mechanical design and mounting of multiple boards in the rack. The space between two adjacent RF inputs will be 1.5 inches. Besides eight RF $1.3 \mathrm{GHz}$ input channels, the receiver board will have 16 down converted IF $(13 \mathrm{MHz})$ output ports, 1 input LO $(1.313 \mathrm{GHz})$ port and various others $\mathrm{I} / \mathrm{O}$ ports as shown in Figure 1. One receiver board will be equipped with temperature sensors and various testing points in order to facilitate the debugging of the bard or to inject signals in the operational conditions (for instance

*Work supported by Fermi Research Alliance LLC. Under DE-AC02$07 \mathrm{CH} 11359$ with the U.S. DOE

"mavric@fnal.gov dithering). We also plan to mount fault cable detectors and LO power monitors.

\section{ANALYSIS OF MAIN PARAMETERS IN THE RECEIVER}

\section{Broad-band Noise Figure}

Broad-band noise is assumed to be dominated by amplitude noise. The overall noise figure of one channel

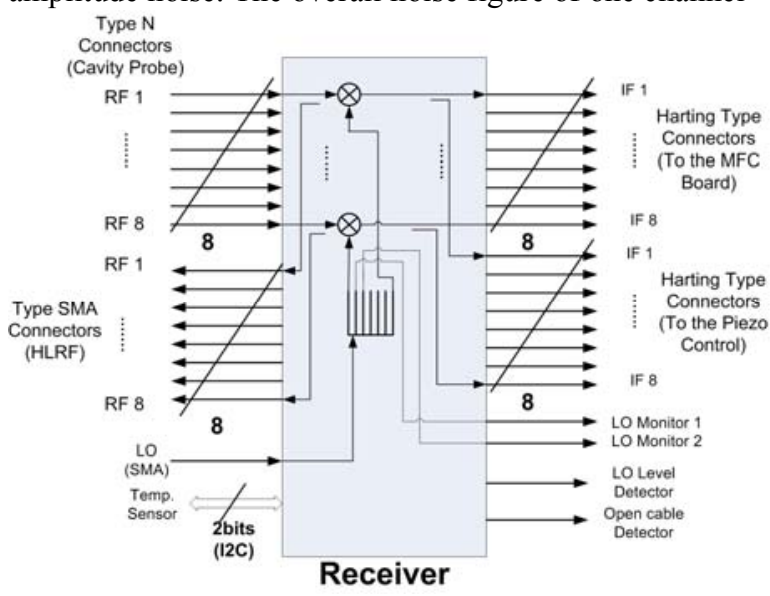

Figure 1: Block diagram of input and output ports of the receiver.

is calculated according to the noise figure values given in datasheets. Figure 2 shows the schematic of one channel and absolute noise power levels at some test points along the processing chain.

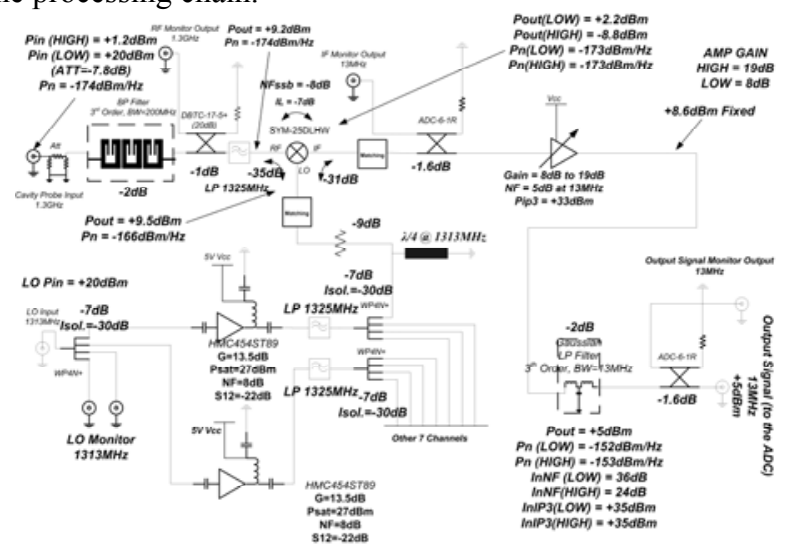

Figure 2: Schematic of one channel. Noise power levels, signal power levels and nonlinearities are shown. 
According to these calculations we expect to achieve app. $-152 \mathrm{dBm} / \mathrm{Hz}$ of noise power at the output, which is equivalent to $36 \mathrm{~dB}$ of input noise figure. The output power level is app. $+5 \mathrm{dBm}$, to match the Multi-channel Field Control module (MFC) [1] input power requirements. The relative noise floor is consequently $157 \mathrm{dBc} / \mathrm{Hz}$. This is much lower than a $-148 \mathrm{dBc} / \mathrm{Hz}$ noise floor of the 12bit ADC on the MFC board and is about the same as a 14bit part. It is supposed that the noise figure of the mixer is app. $1 \mathrm{~dB}$ higher than its insertion loss (see literature [2]) and that we managed to suppress all the mixing products by proper matching. Broad-band amplitude noise introduced by the LO is supposed not to affect the overall noise floor since the LO amplifiers are driven in saturation and the LO signal is filtered. The main limiting component for the schematic in Figure 2, in terms of broad-band noise floor, is the IF amplifier. If lower-broad band noise specifications are to be met, a higher input signal and a more linear mixer can be used. In this case the IF amplifier can have lower gain. However, higher mixer linearity is usually associated with higher LO port power, which means that the LO amplifier has to be higher gain. This contributes to a higher close in phase noise as presented in the following paragraph. The design presented in Figure 2 is a compromise between linearity, broad-band noise, close in phase noise and LO input power.

Table 1: Equivalent time rms jitter and phase rms deviation for some mixers presented in Figure 3. (Integration bandwidth $100 \mathrm{~Hz}$ to $500 \mathrm{kHz}$ )

\begin{tabular}{|l|c|c|}
\hline Mixer & $\mathbf{t}_{\text {jrms }}[\mathbf{f s}]$ & $\boldsymbol{\varphi}_{\mathbf{j r m s}}\left[^{\circ} \mathbf{]}\right.$ \\
\hline HMC483MS8G (Active) & 2.4 & $1.1 \mathrm{e}-3$ \\
\hline SYM25DLHW (L10) & 1.6 & $7.8 \mathrm{e}-4$ \\
\hline ZFM-2000 (L7) & 1.8 & $8.6 \mathrm{e}-4$ \\
\hline SYM25DHW(L17) & 1.4 & $6.7 \mathrm{e}-4$ \\
\hline
\end{tabular}

\section{Narrow-band Noise Figure}

Narrow-band noise figure of the receiver is assumed to be defined only by phase noise. Phase noise introduced in the LO distribution of the receiver is translated to the IF port and it is defined by the slope of the mixer (V/rad) (for more details see [3]). The major phase noise contributor in the LO distribution part of the receiver is the LO amplifier. The phase noise issues of a microwave amplifier are thoroughly studied in literature [4]. Input power to the LO amplifier and the nonlinearity of the amplifier define the amount of the induced flicker noise. The flicker noise is up-converted to the carrier, which is transmitted through mixing on the IF port and therefore to the whole system. Figure 3 shows an example of the amount of phase noise generated by an MMIC amplifier (HMC481MP86, www.hittie.com). The measurement method used for measurements presented in Figure 3 is known as single oscillator phase noise measurement technique according to literature [5]. The results in Figure 3 show that in the case of these particular mixers it is better to use a passive mixer with an external LO amplifier. It also shows that we do not need to drive a higher level mixer to get the best phase noise performances. However, lower LO power decreases linearity, which is investigated in the following section. The integrated phase noise for various mixers is given in Table 1. The measurement method used for measurements presented in Figure 3 is known as single oscillator phase noise measurement technique according to literature [5]. The integrated phase noise for various mixers is given in Table 1 .

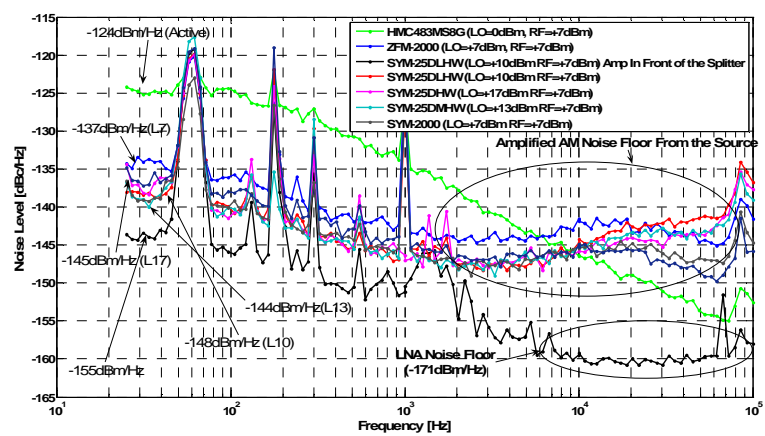

Figure 3: Close in phase noise measurements for mixer and LO amplifier combination.

\section{Nonlinearities}

A lower level mixer is usually less linear than a higher level mixer. Measurements of mixers presented in Figure 3 would show that there is approximately $20 \mathrm{~dB}$ difference between the power in the second and the third harmonic for the level 7 and the level 17 mixers. Measurements show that level 13 and level 10 mixers exhibit very similar linearity performance, which favorizes the level 10 mixer. Comparison of a level 10 mixer and level 17 mixer shows that the second and the third harmonic differ for approximately $13 \mathrm{~dB}$ between the two mixers. For the receiver design we have chosen the level 10 mixer due to lower LO amplifier gain requirements.

A perturbation analysis with the 8 channel receiver and 8 superconducting RF cavities was carried out. Dispersion in peak gradients between cavities causes the calibrated channels in the receiver to work at different points of the nonlinear curve. Analysis shows that the receiver should operate in the compression point region between $0.01 \mathrm{~dB}$ and $0.1 \mathrm{~dB}$ if less than $0.1 \%$ of gradient deviation wants to be achieved.

\section{Cross-Talk between RF Channels}

In order to minimize cross-talk between channels special attention has to be paid to cables, layout, chassis design and matching of components. The IF cables connecting the receiver chassis and the MFC digital board are manufactured by Harting and measurements show that isolation between adjacent channels is better than $80 \mathrm{~dB}$. One of the most critical parts in terms of matching and isolation is the mixer. There are various paths through the mixer that cause the RF or IF signal to couple in the adjacent channel. Measurements show that there is a 
difference of more than $10 \mathrm{~dB}$ between a poorly matched mixer and a mixer with an improved match. Besides matching and cables, proper layout and chassis construction (shielding) is also an important way of coupling reduction.

\section{Temperature Stability of the Receiver}

A receiver has been built on an aluminium plate with heaters and temperature sensors regulated by a temperature controller. Temperature excursions of $2^{\circ} \mathrm{C}$ on the aluminium plate were induced and a phase fluctuation that equals to $0.25^{\circ}$ was measured. Using a more sophisticated temperature controller (PID) would decrease large temperature excursions and consequently decrease phase deviation swings.

\section{IMPLEMENTATION OF THE 8 CHANNEL RECEIVER}

\section{One Channel Layout}

The specific parts that are planned to be used for the 8 channel receiver board are shown in Figure 2. The final choice of IF amplifier has not yet benn made. Analog Devices and other manufacturers offer a broad spectrum of ultra low noise variable gain amplifiers. However, they are designed to drive high impedance loads $(>500 \Omega)$, which causes additional power losses in impedance transformers. At higher gains the nonlinearity becomes a serious issue. Monolithic microwave integrated circuit amplifier modules are easier to use but tend to be more nonlinear and are usually meant to be used at higher frequencies.

\section{Eight Channel Layout}

Figure 4 shows a design of the bottom and top layer of the board. Each board will be $8 \mathrm{U}$ (14 inches) high. The input down-converter modules will be enclosed in a aluminium cage. Isolation between LO branches will be reinforced by ground fences and proper line routing.

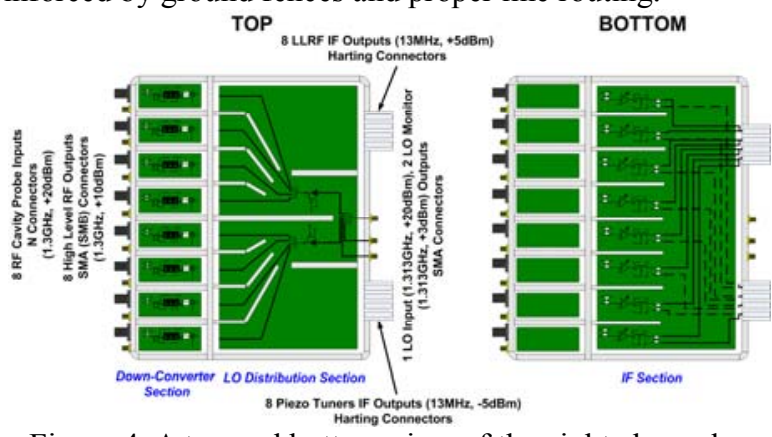

Figure 4: A top and bottom view of the eight channel receiver board.
Each of 12 8-channel boards will be placed on the top of the rack in a crate. The crate will be slightly tilted so that the rigid cables will not need to be additionally banded before coming to the receiver board.

\section{MEASUREMENT RESULTS OF THE PROTOTYPE}

According to the schematic in Figure 1, a two channel prototype was constructed. Before acquisition, the two $13 \mathrm{MHz}$ signals were phase shifted in order to be in quadrature. The acquired digital data was multiplied and filtered with a $500 \mathrm{kHz}$ low-pass FIR filter. This gives the residual phase noise of the receiver and MFC board. Any common phase deviation of the source generator is subtracted by the method. Standard deviation of the filtered signal is $3 \mathrm{e}-3^{\circ}$ at $500 \mathrm{kHz}$ SSB bandwidth.

On the IF port of the prototype we measured the second and the third harmonics produced by the mixer, which were $40 \mathrm{~dB}$ high and the isolation between these two channels was approximately $88 \mathrm{~dB}$ with proper matching on the mixer ports.

\section{CONCLUSIONS}

In the paper we discussed the main parameters that have to be considered for proper operation of the receiver. A target value for broad-band noise at the output of the receiver is $-157 \mathrm{dBc} / \mathrm{Hz}$, the integrated close in phase noise over at $500 \mathrm{kHz}$ bandwidth should be better than $3 \mathrm{e}-3^{\circ}$, the receiver should operate between $0.01 \mathrm{~dB}$ and $0.1 \mathrm{~dB}$ compression point and cross-talk measurements show that we can achieve better than $80 \mathrm{~dB}$ of isolation. A per se part of the project is the implementation where mechanics of the chassis, ease of module access and diagnostics have to be considered. At present we are in the phase of 8 channel board layout and two channel prototype testing.

\section{REFERENCES}

[1] Varghese P., Barnes B., Branlard J., Chase B., W Joireman P., Klepec D., Mavric U., Tupikov V., "Multichannel Vector Field Control Module for LLRF Control of Superconducting Cavities", This Conference - WEPMN112

[2] A. Mass S. , "Microwave Mixers - Second Edition", Artech House, Inc. 1993

[3] Walls F.L. and Stein S.R., "Accurate Measurements of Spectral Density of Phase Noise in Devices", National Bureau of Standards

[4] Hati A., Howe D.A., Walls L.F., Walker D.K.,"Merits of PM Noise Measurements over Noise Figure: A Study at Microwave Frequencies", IEEE Transactions on Ultrasonics, Ferroelectrics and Frequency Control, vol.53, No.10, October 2006

[5] "Infrared and Millimeter Waves", Vol.11, pp.239289,1984 